\title{
5
}

\section{ICE GRAINS IN SPACE}

\author{
R. SMOLUCHOWSKI
}

Certain astrophysically interesting properties of ice grains depend upon their crystallinity. Lower temperatures such as those in the outer layers of a dust cloud favor amorphous ice, the center of the cloud may favor crystalline ice. Amorphous grains have different binding energies than crystalline grains resulting in different evaporation rates and affecting the distance from the sun at which dust is released in cometary tails. They have also different sticking and accommodation coefficients which affect the formation of $\mathrm{H}_{2}$ and of other molecules on their surfaces. In contrast to crystalline grains, amorphous grains can be easily ionized by the stellar ultraviolet photons with important consequences for surface reactions, heating, etc.

1. Many properties of the interstellar medium can be explained by assuming the existence of grains of various solids such as graphite and water ice and a great deal of research has been devoted to this topic. These considerations are usually based on the assumption that these materials are simply small grains of the common terrestrial solids. Within the last few years, however, great progress has been made -- both in theory and in experiment - - concerning the physics and chemistry of small grains and of solids formed by slow condensation at low temperatures. Inasmuch as some of their properties differ considerably from those of the normal bulk solids they may play a significant role in the interpretation of astrophysical observations. Admittedly not all of the recent research deals specifically with ice but many conclusions can be based on analogies with other solids and with theoretical models. The purpose of this paper is to outline these new aspects of ice grains without going into detailed evaluations.

2. The sequence of ices obtained during slow deposition such as occurs in interstellar space is approximately as follows: at $10^{\circ} \mathrm{K}$ amorphous ice $I_{A 1}$ is formed with a density around $1.2 \mathrm{~g} \mathrm{~cm}^{-3}$ (Venkatesh et al. 1974) and this form persists up to $77^{\circ} \mathrm{K}$; at temperatures up to about $140^{\circ} \mathrm{K}$ another amorphous ice $\mathrm{I}_{\mathrm{A} 2}$ is obtained (F1etcher 1970) with a density $0.93 \mathrm{~g} \mathrm{~cm}^{-3}$ which on heating transforms irreversibly into cubic crystalline ice $I_{c}$ of about the same density. At still higher temperatures the usual stable hexagonal crystalline ice Ih is formed with a density of $0.92 \mathrm{~g} \mathrm{~cm}^{-3}$. It should be stressed that the sometimes considerable differences between temperature limits quoted by various authors are usually due to different rates of deposition of these metastable forms. The range of temperatures here quoted encompasses those assumed to exist in various parts of space where grains are formed or exist. Since in a dust cloud the temperatures may vary by a factor of ten between the center and the outer layers (Leung 1975) one may expect a radial gradient of ice grains (or ice covered grains) of different structures and properties. 
3. In parallel with the variation of structure one expects a considerable variation in the average binding energy of an $\mathrm{H}_{2} \mathrm{O}$ molecule to the grain. $\mathrm{Al}-$ though no quantitative information on this subject for ices seems to be available one expects the amorphous ice $I_{A 2}$ to have (Briant and Burton 1975) up to 25 percent fewer hydrogen bonds than the crystalline form and thus a correspondingly weaker binding. On the other hand the $I_{A 1}$ form because of its higher density should have a binding which is stronger by a comparable amount. These differences will affect for instance the rates of sublimation of these grains and the critical distance from the sun at which grains in cometary tails break up and release dust and impurities (Sekanina 1975).

4. It is known that slow growth of a cluster of atoms can lead to a progressively looser structure so that the number of nearest neighbors, the binding and the density are lower near the outside than in the center (Bennett 1972). This difference could be as high as 40 or 50 percent. If these results apply to amorphous ice they could affect their optical properties (Smoluchowski 1977) and the sticking and accommodation coefficients. The sticking coefficient $S$, that is the fraction of incident atoms which becomes thermalized and permanently attached to the grain, would be lower because of weaker binding to the surface. and lower loss of energy on each jump (Hollenbach and Salpeter 1970). One can expect this effect to be roughly proportional to the change in the number of nearest neighbors as compared to crystalline ice. The accommodation coefficient $\alpha$, that is the fraction of the energy of the atom transferred to the grain, will be similarly affected. In the range of existence of ice $I_{A 1}$ the coefficients $S$ and $\alpha$ would be higher than for the normal ice $I_{h}$ : by a few percent below $10^{\circ} \mathrm{K}$, by 12 percent near $20^{\circ} \mathrm{K}$ and by 50 percent near $50^{\circ} \mathrm{K}$. For ice $\mathrm{I}_{\mathrm{A} 2}$ these coefficients would be lower by similar amounts. No major differences would be expected for $i$ ces $I_{c}$ and $I_{h}$. The effect of these altered coefficients $S$ and $\alpha$ could be pronounced for the rates of formation of interstellar $\mathrm{H}_{2}$ from $\mathrm{H}$ atoms (Hollenbach and Salpeter 1971) on the surfaces of grains and for other surface processes:

5. Interstellar flux of ultraviolet photons leads to photoionization of grains which become positively charged. The long range of the electrostatic force as compared to van der Waals attraction increases the probability of capturing a neutral atom and decreases this probability for a positive ion. Therefore it plays an important role in the formation of various molecules (Field 1974). The emission of photoelectrons is also an important mechanism for heating the dust cloud and for giving the grains momentum. Normally photoionization of ice grains (or ice coated grains) is considered unimportant (Watson 1972) because the ionization threshold lies close to $13.5 \mathrm{eV}$ which happens to coincide with the drop of intensity of the interstellar spectrum. The latter is essentially flat in wavelength down to about $10 \mathrm{eV}$ (Habing 1968) that is the number of photons is proportional to $v^{-1}$. It is known that amorphous solids have a narrower band gap and a band edge which is shifted to lower frequencies than crystalline solids; it follows that their photoionization threshold should lie at lower photon energies. Recent data (Baron and Williams 1976) suggest that the threshold for ice $I_{A 2} 1$ ies between 8 and $9 \mathrm{eV}$. This result indicates that contrary to earlier conclusions ice grains (or ice coated grains) will be easily ionized by the abundant ultraviolet flux in the $10-13.5 \mathrm{eV}$ range. This will be particulary true for ice $I_{A 1}$. It follows that ice grains should play a significant role in the processes mentioned at the beginning of this section.

A more detailed presentation of the various estimates and of the astrophysical consequences will be published elsewhere (Smoluchowski 1977).

\section{REFERENCES}

Baron, B., and Williams, F. 1976, J. Chem. Phys., 64, 3896. 
Bennett, C. H. 1972, J. Appl. Phys., 43, 2727.

Briant, C. L., and Burton, J. J. 1975, J. Chem. Phys., 63, 3327.

Field, G. B. 1974, Highlights of Astronomy, 3, 37.

Fletcher, N. H. 1970, The Chemical Physics of Ice, Cambridge, U. Press, Cambridge.

Habing, H. J. 1968, Bu11. Astr. Inst. Netherlands, 19, 421.

Hollenbach, D., and Salpeter, E. E. 1970, J. Chem. Phys., 53, 79.

Hollenbach, D., and Salpeter, E. E. 1971, Ap.J., 163, 155.

Leung, C. M. 1975, Ap.J., 199, 340 .

Sekanina, Z. 1975, Icarus, 25, 218.

Smoluchowski, R. 1977, (in preparation).

Venkatesh, C. G., Rice, S. A., and Narten, A. H. 1974, Science, 186, 927.

Watson, W. D. 1972, Ap.J., 176, 103.

\section{DISCUSSION}

WHIPPLE: Do you predict that a grain made of amorphous ice I would carry a larger positive charge in a radiation field than other $k$ inds of ice grains?

SMOLUCHOWSKI: I would rather say that that the ionization of amorphous ice $I$ is much more probable than for other ices. Thus for a given shape of a grain and for a particular flux of photons of energy hv its charge should be higher.

DELSEMME: In collaboration with Wenger, we have inadvertently discovered this Amorphous Ice $I$ of density 1.2 in 1969, for which we had erroneously reported a density much larger than 1.2, but we also found that it disappeared in presence of extremely small amounts of impurities, and concluded that it could not have any significance in cometary physics. Ref.: Delsemme, $A . H$. , and Wenger, $A$. 1969, Planet. Space Sci., 18, 709. 